\title{
La infiltración con corticoides alivia más rápidamente el hombro doloroso rígido que la fisioterapia.
}

Effectiveness of corticosteroid injections versus physiotherapy for treatment of painful stiff shoulder in primary care: randomized trial. D. A.Van der Windt, B. W. Koes, W. Devillé, A. J. P. Boeke, B. A. de Jong, L. M. Bouter. BMJ 1998;317:1292-6.

\section{Objetivo}

Comparar la efectividad de las inyecciones con $40 \mathrm{mg}$ de acetonida de triamcinolona vs fisioterapia, para el tratamiento de hombro doloroso rígido (hombro congelado o capsulitis adhesiva).

\section{Diseño}

Ensayo clínico aleatorizado simple ciego.

Lugar

Centros de salud en Amsterdam, Holanda.

\section{Pacientes}

Se incluyeron 109 pacientes, mayores de 18 años, que consultaron a sus médicos de cabecera por dolor de hombro y presentaban restricción de la movilidad glenohumeral. No debían presentar síntomas bilaterales, ni haber recibido tratamiento con corticoides o kinesioterapia en los últimos seis meses. Se excluían clínicamente los dolores de hombro periarticulares (bursitis subacromial, tendinitis).

\section{Intervención}

Se asignó a los pacientes, en forma aleatorizada, a dos grupos de tratamiento con una duración total de 6 semanas cada uno. Uno consistía en realizar 12 sesiones de 30 minutos de movilización articular pasiva y ejercicios del hombro. El otro en inyecciones en la articulación gleno-humeral, realizadas por médicos de familia entrenados, con 40 $\mathrm{mg}$ de acetonida de triamcinolona (realizando un promedio de 2,2 infiltraciones). El seguimiento de los pacientes fue de un año.

\section{Medición de resultados principales}

Los resultados fueron evaluados a las 3, 7, 13, 26 y 52 semanas. El resultado principal fue definido como la mejoría percibida por el paciente, objetivada mediante una escala de seis puntos (escala de Likert). Para evaluar una tasa de éxito se preguntó al paciente si sentía una mejoría muy importante o recuperación total, y si esto ocurría se consideró un éxito. Los pacientes fueron interrogados con un score de doLor asociado a su molestia principal. La severidad del dolor durante el día y la noche fue consignada en una escala analógica visual (100 era un dolor muy severo y 0 no dolor), mientras que la dificultad funcional del miembro afectado fue evaluada mediante un cuestionario de situaciones comunes que producen dolor. El resultado secundario era el grado de restricción de movilidad pasiva en abeducción del miembro superior y la rotación externa, comparando con el miembro sano y usando un inclinómetro digital.

\section{Resultados principales}

El análisis por intención de tratar mostró que a las 7 semanas de tratamiento, el $77 \%$ (40 de 52 pacientes) tratados con infiltraciones tuvieron una mejoría clínicamente significativa comparado con $46 \%$ (26 de 56 pacientes) tratados con kinesioterapia. La diferencia absoluta entre los dos grupos fue de $31 \%$ (IC 95\% 14 a $48 \%$ ) y el NNT de 5 (IC $95 \% 2$ a 7). Esta diferencia fue significativa para todos los resultados, excepto para la restricción en la abeducción. En la evaluación de las 26 y 52 semanas la diferencia entre los dos grupos fue pequeña (no informaron los valores). Los efectos adversos en ambos grupos fueron leves. El grupo kinesioterapia requirió mayor cantidad de tratamiento extra (paracetamol, inyección con corticoide, kinesioterapia) que el grupo infiltración. Hubo una buena tasa de seguimiento de los pacientes.

\section{Conclusiones}

Este estudio demuestra que, en los pacientes que sufren de hombro doloroso rígido, la infiltración articular alivia más rápidamente el doLor y la abeducción del hombro que el tratamiento con kinesioterapia. En el seguimiento al año del tratamiento esta diferencia se hace más pequeña (no reporta significancia).

\section{COMENTARIO}

Este trabajo es muy interesante ya que demostró que el tratamiento con inyecciones de corticoides intraarticulares, realizado por médicos de familia entrenados, es superior a la kinesioterapia eń el tratamiento del hombro rígido o congelado a las 7 semanas. La ventaja del tratamiento con inyecciones es la rapidez de alivio de los síntomas. Es importante destacar que en el análisis del resultado principal fue incorporada una variable categórica, la mejoría o no, percibida por el paciente. Esto es clínicamente razonable y puede trasladarse a la práctica de una forma más confiable que los otros resultados evaluados con las escalas numéricas, pero también es un punto observable ya que el estudio fue realizado a simple ciego (ya que las intervenciones eran muy diferentes), lo cual podría sesgar algunas respuestas si los participantes perciben al tratamiento invasivo como más eficaz.
Es importante recordar que el dolor de hombro rígido o "congelado" no es el más frecuente de ver en atención primaria. En cambio es común la periartritis de hombro, que se presenta como dolor a la movilidad sin restricción, que también puede ser tratada con kinesioterapia e inyecciones locales con corticoides, aunque hasta ahora los resultados de los estudios realizados no son concluyentes a favor 0 en contra de la infiltración. ${ }^{1}$ Las infiltraciones pueden aliviar también el dolor de diversos problemas de salud como epicondilitis, sdme. del túnel carpiano, bursitis trocantérea, bursitis anserina (o tendinitis de pata de ganso), entre otras. Considero que esta estrategia terapéutica puede ser realizada fácilmente por los médicos de atención primaria una vez entrenados (tal como ocurre en países como Canadá o EEUU), constituyendo una herramienta efectiva y de muy bajo riesgo para tratar a nuestros pacientes.

Dr. Ricardo Frusso

Unidad de Medicina Familiar y Preventiva. Hospital Italiano de Buenos Aires. 